Supporting Information

\title{
Neutrophil-Like Cell Membrane-Coated Nanozyme Therapy for Ischemic Brain Damage and Long-Term Neurological Functional Recovery
}

Lishuai Feng ${ }^{1 \neq}$, Chaoran Dou ${ }^{1 \neq}$, Yuguo Xia ${ }^{1+}$, Benhao Li ${ }^{2}$, Mengyao Zhao ${ }^{2}$, Peng Yu ${ }^{2}$, Yuanyi, Zheng ${ }^{1}$, Ahmed Mohamed El-Toni ${ }^{3,4}$, Nada Farouk Atta ${ }^{5}$, Ahmed Galal ${ }^{5}$, Yingsheng Cheng ${ }^{1 *}$, Xiaojun Cai ${ }^{1 *}$, Yan Wang ${ }^{1 *}$, Fan Zhang ${ }^{2 *}$

${ }^{1}$ Department of Radiology, Department of Ultrasound in Medicine, Department of Neurosurgery, Shanghai Jiao Tong University Affiliated Sixth People's Hospital, Shanghai 200233, P. R. China.

${ }^{2}$ Department of Chemistry, State Key Laboratory of Molecular Engineering of Polymers and Shanghai Key Laboratory of, Molecular Catalysis and Innovative Materials and iChem, Fudan University, Shanghai 200433, P. R. China

${ }^{3}$ King Abdullah Institute for Nanotechnology, King Saud University, Riyadh 11451, Saudi Arabia

${ }^{4}$ Central Metallurgical Research and Development Institute (CMRDI), Helwan, 11421 Cairo, Egypt

${ }^{5}$ Department of Chemistry, Cairo University, Giza, 12613 Cairo, Egypt

$\$$ These authors contributed equally

\section{*Corresponding authors}

E-mail: chengyingsheng@hotmail.com; c1x2j34@163.com; yannanfly@126.com; zhang_fan@fudan.edu.cn 


\section{Supplementary Figures}

a

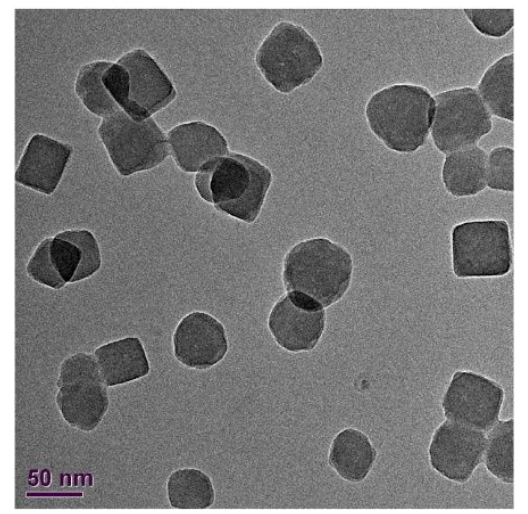

b

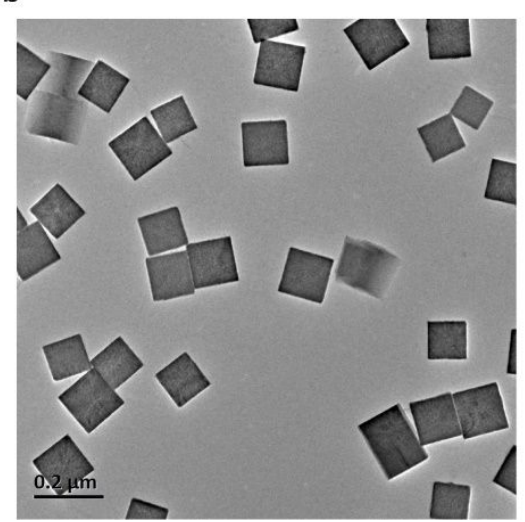

C

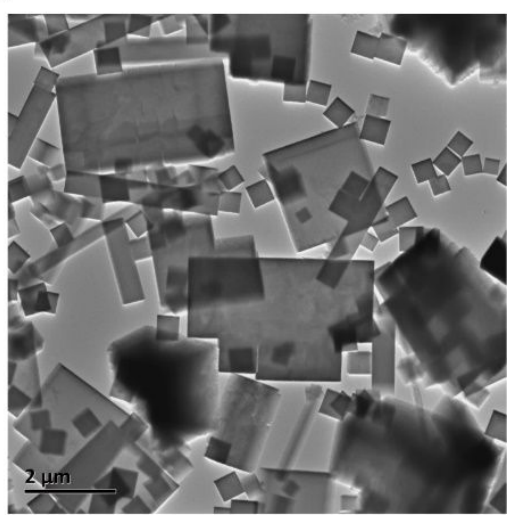

Figure S1. The TEM images of Prussian blue particle generated in $\mathrm{H}^{+}$concentration of (a) 2 $\mathrm{moL} / \mathrm{L}$, (b) $0.01 \mathrm{moL} / \mathrm{L}$ and (c) neutral solution.

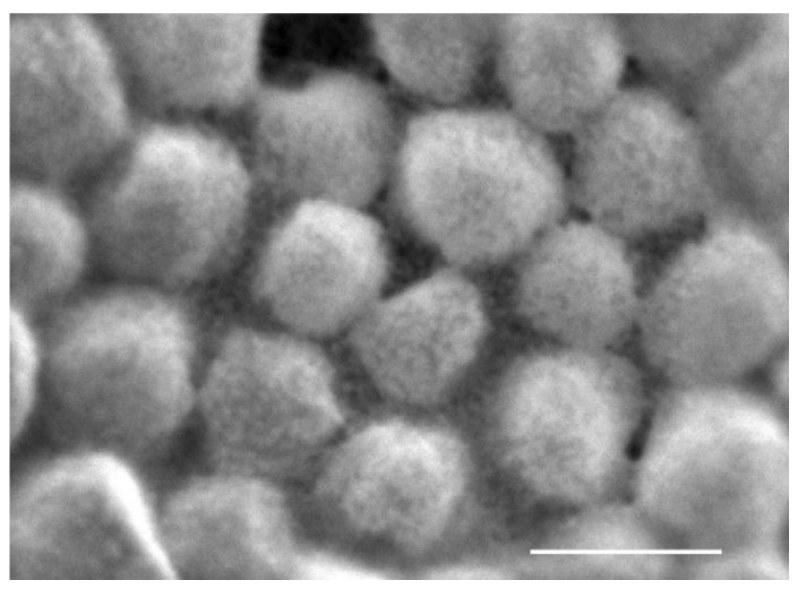

Figure S2. Scanning electronic microscopy (SEM) of MPBzyme. Scale bar: $100 \mathrm{~nm}$.

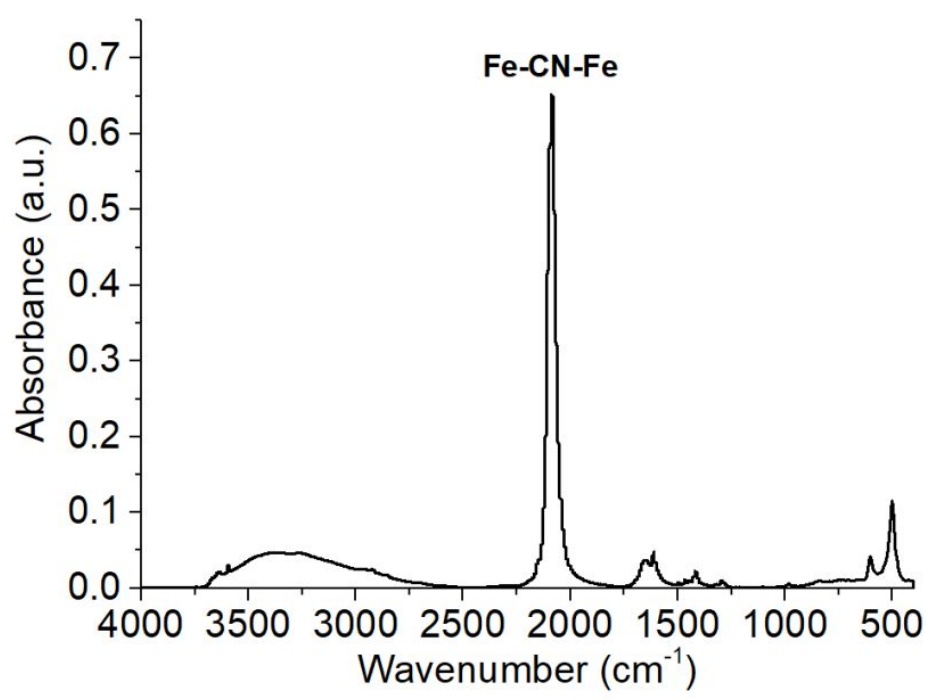


Figure S3. FTIR spectra of MPBzyme.
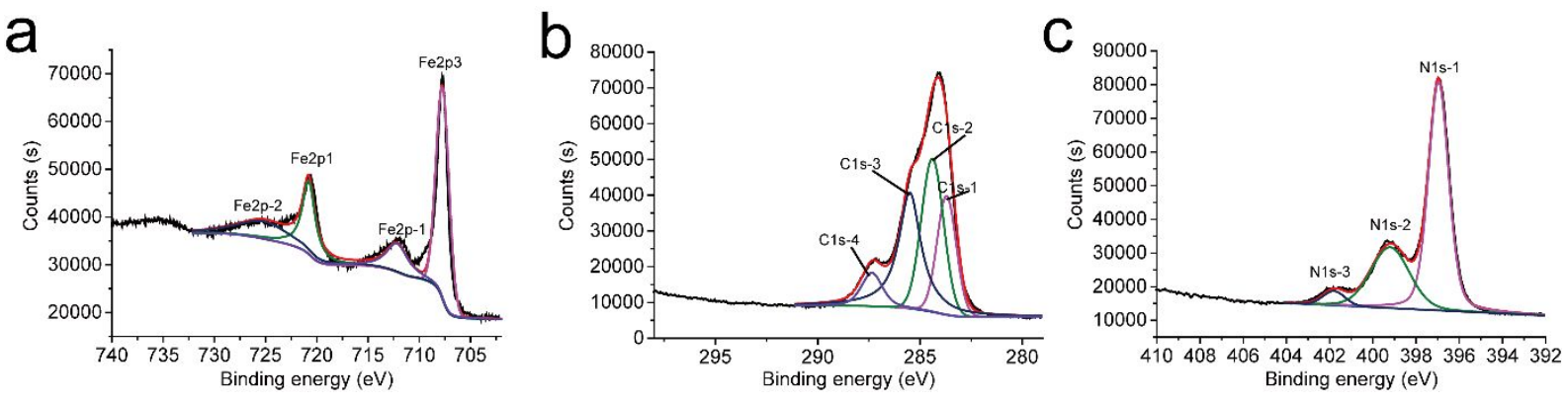

Figure S4. X-ray photoelectron spectroscopy of MPBzyme.

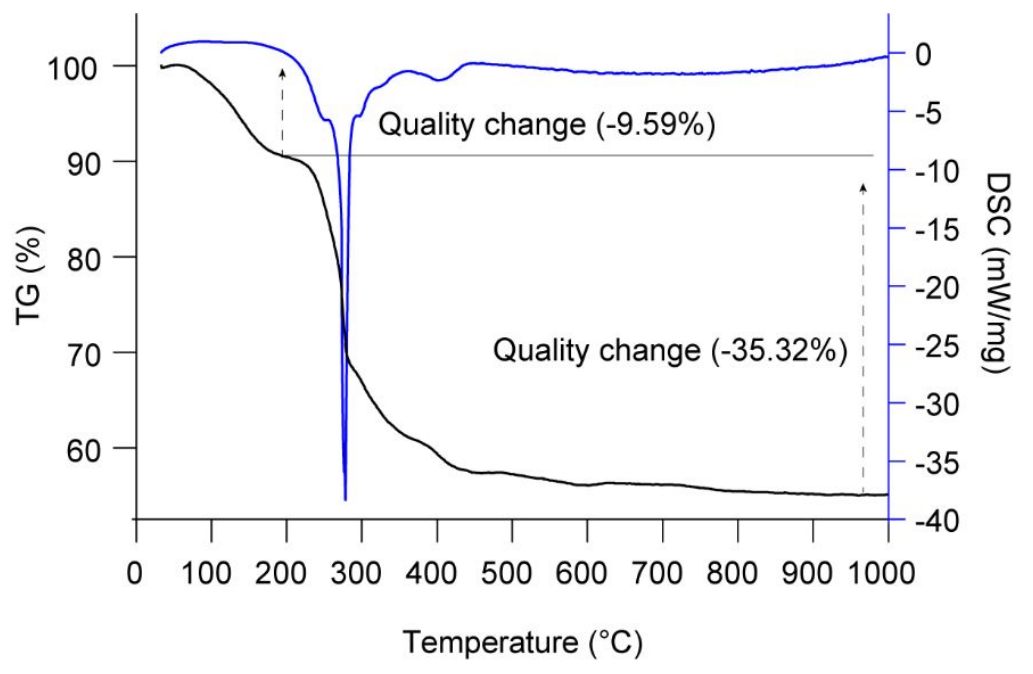

Figure S5. Thermogravimetry-differential scanning calorimetry (TG-DSC) analysis of MPBzyme. 


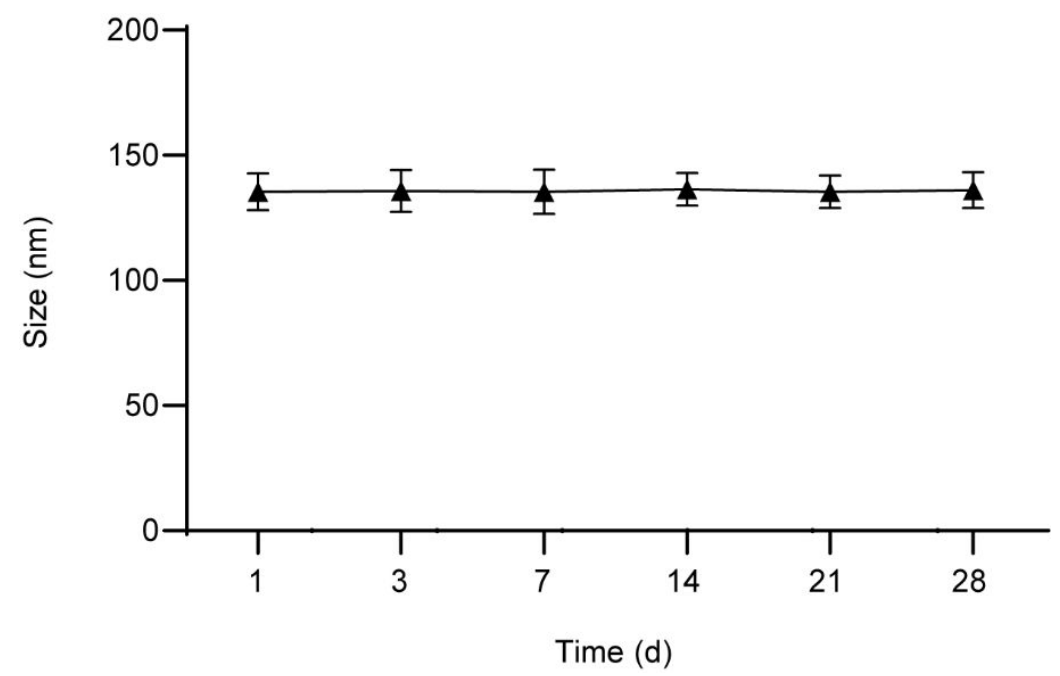

Figure S6. Hydrodynamic diameter of MPBzyme in serum within 28 days.

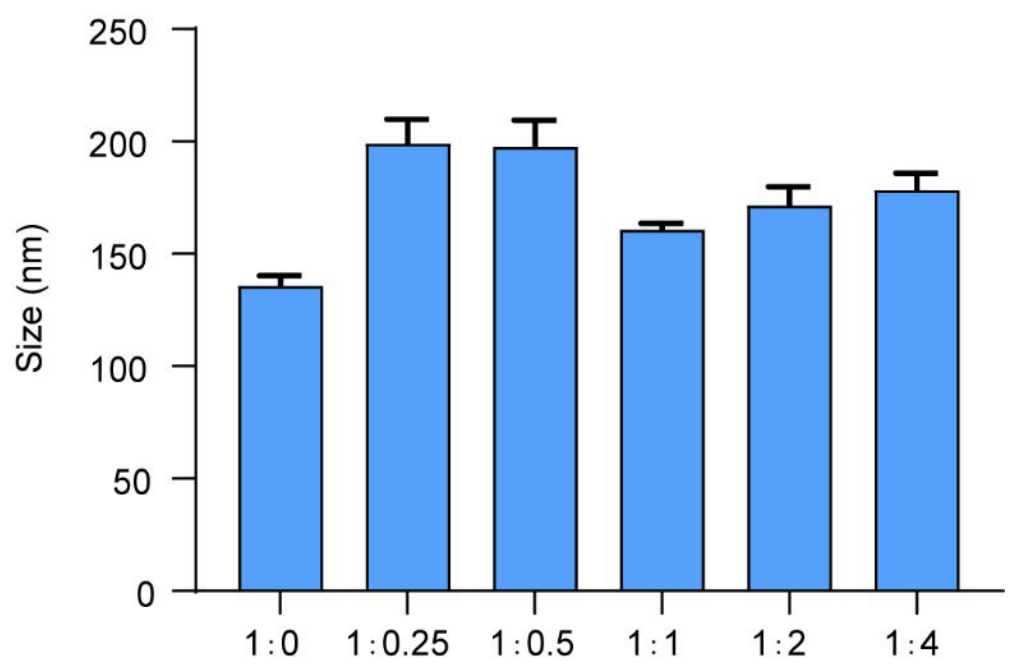

Figure S7. Hydrodynamic size measurements of differentiated HL-60 cells coated MPBzyme with various weight ratios of MPBzyme-to-membrane in saline. 


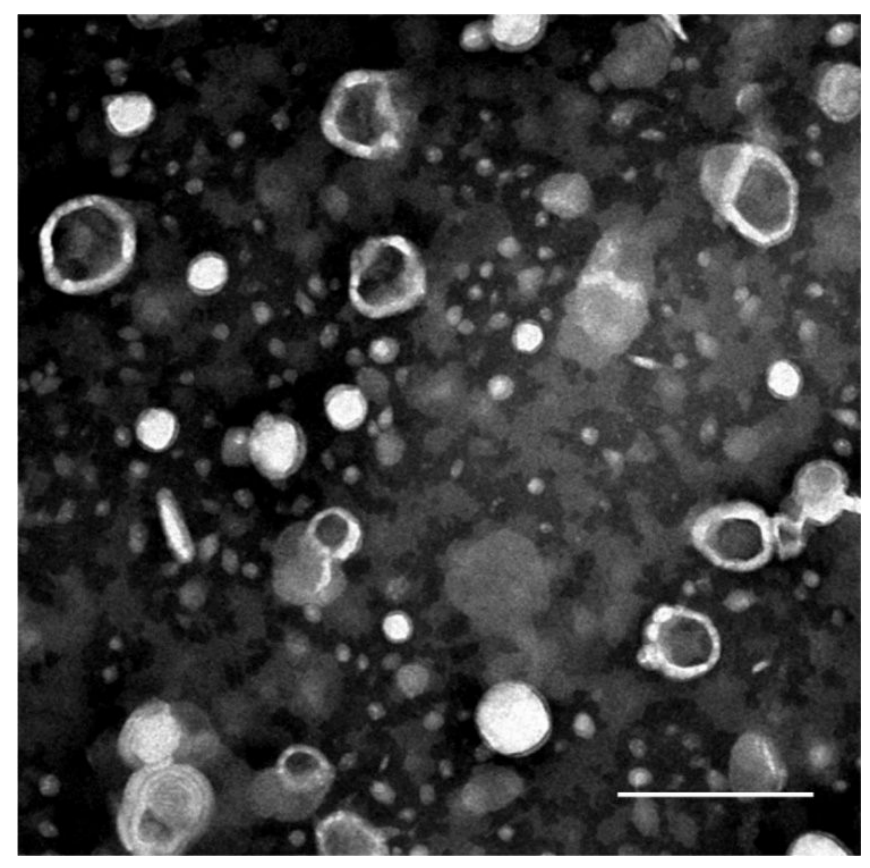

Figure S8. TEM image of MPBzyme@NCM. Scale bar: 200 nm.

a

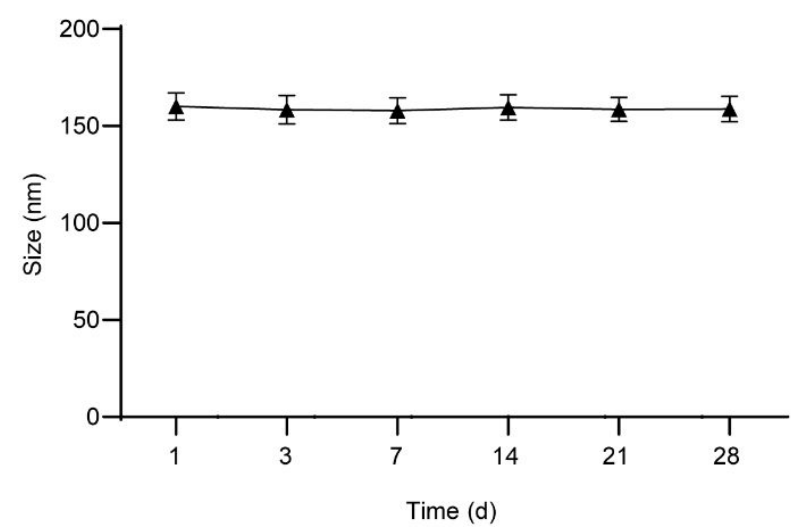

b

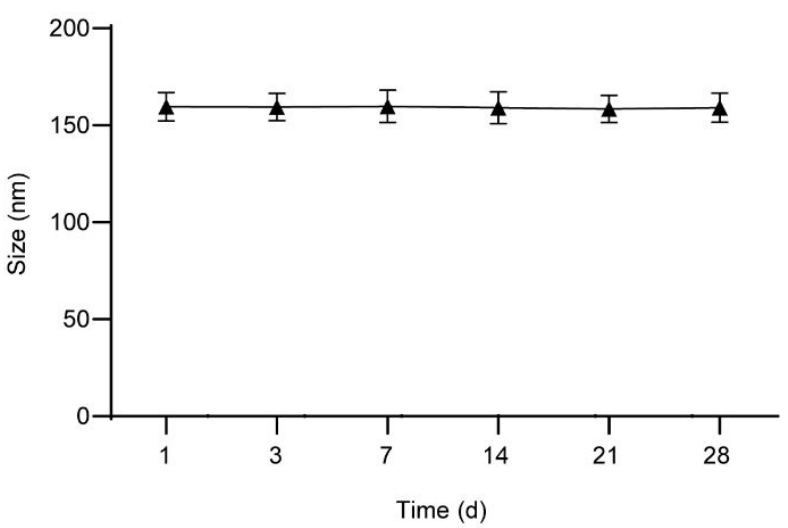

Figure S9. Hydrodynamic diameter of MPBzyme@NCM in (a) saline and (b) serum within 28 days. 


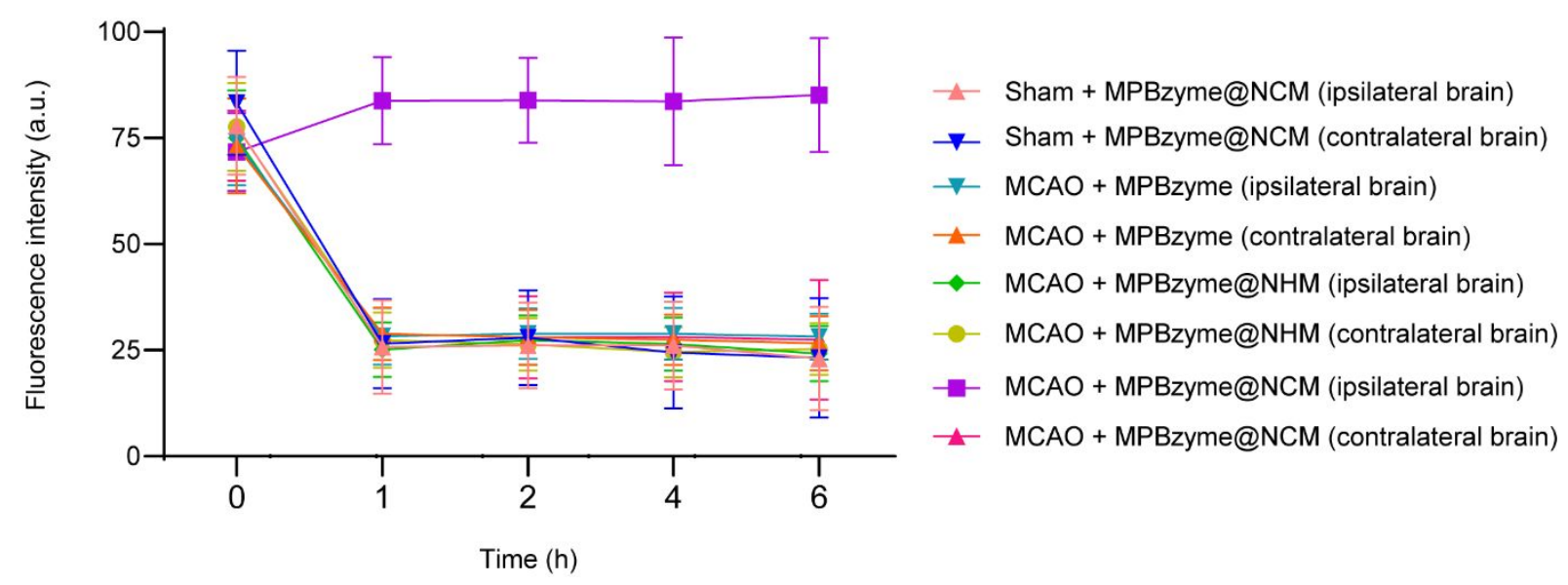

Figure S10. The mean fluorescence intensity of in vivo fluorescence signals within $6 \mathrm{~h}$ in the brains of different-treated mice.

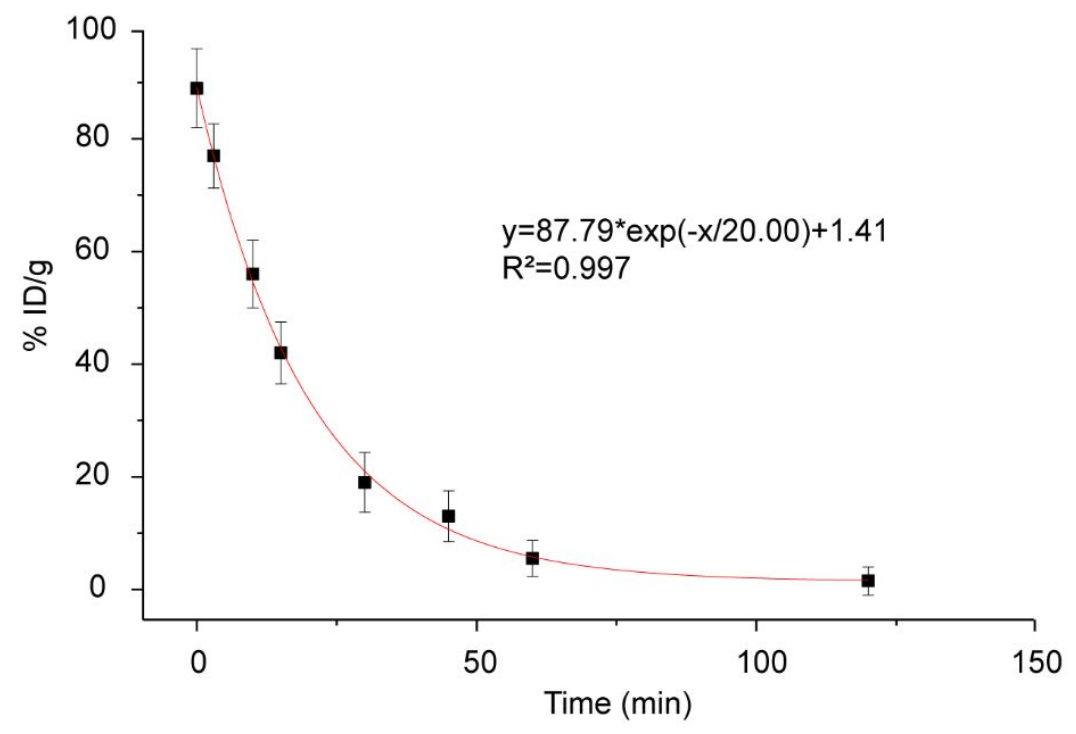

Figure S11. Half-life of MPBzyme@NCM in blood. Data are presented as mean \pm SEM. n=3 per group. 


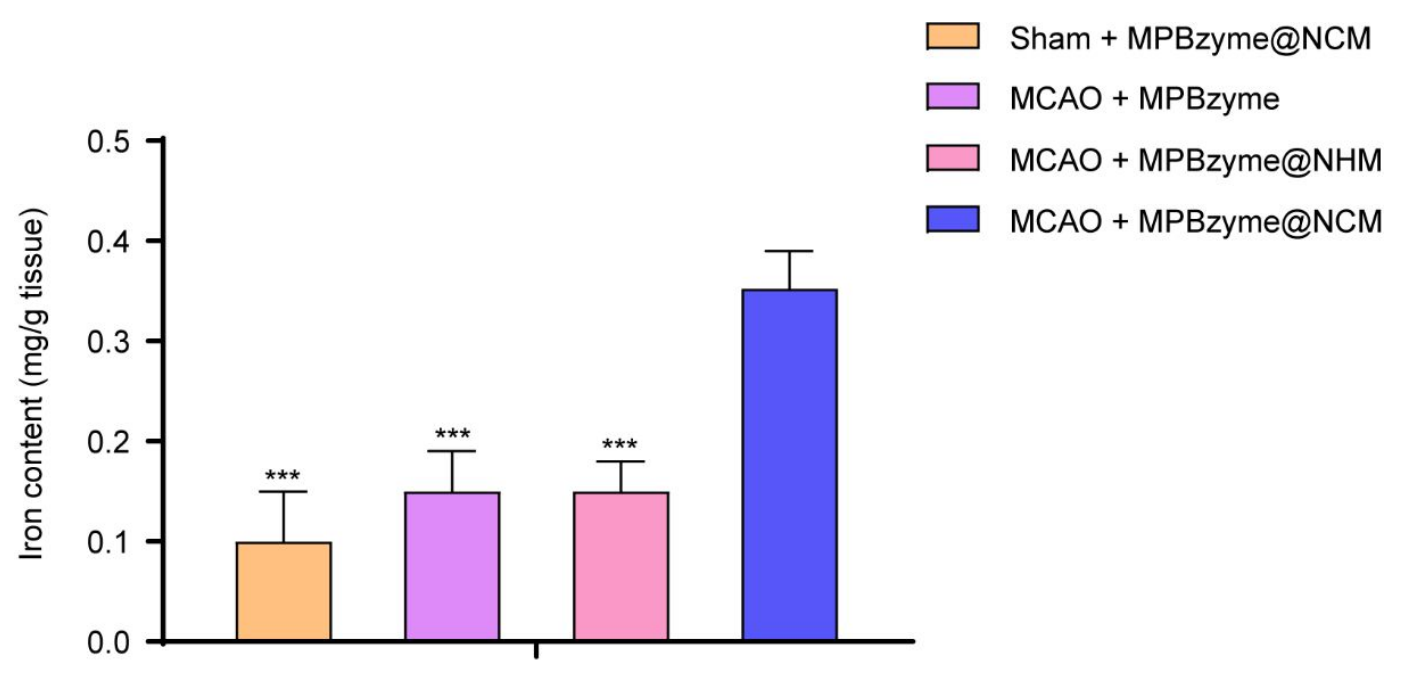

Figure S12. The iron content of brains of mice after different treatment examined by ICP-OES at $24 \mathrm{~h}$ after injection. $\mathrm{n}=3$ per group. Data are presented as mean $\pm \mathrm{SEM}$. P values were calculated by one-way ANOVA (*** P < 0.001, v.s. MCAO + MPBzyme@NCM).

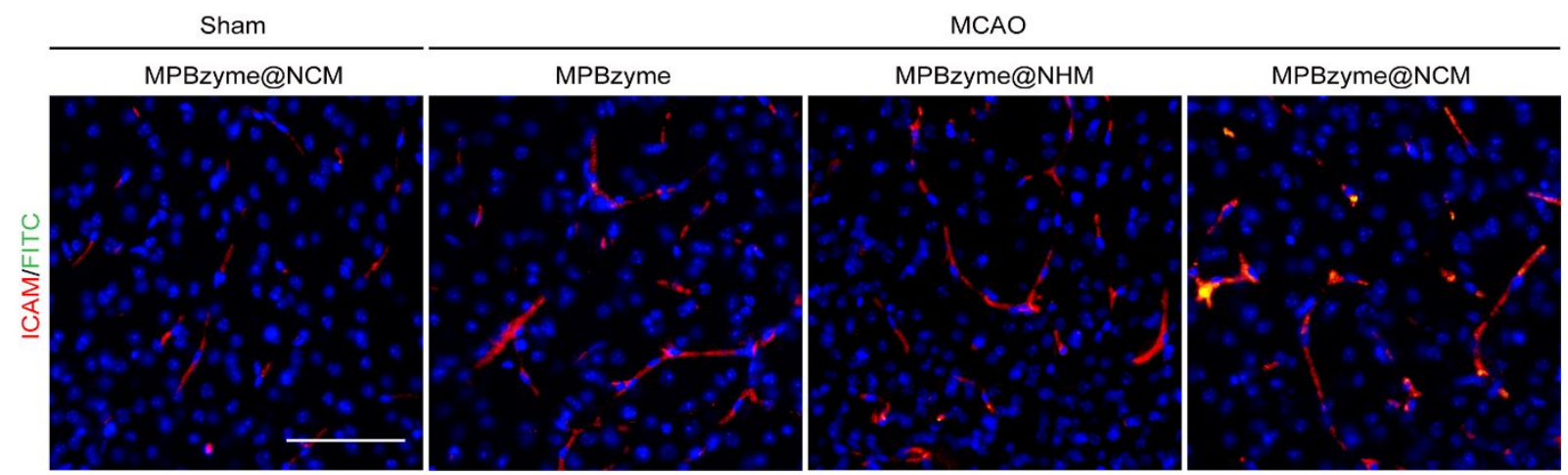

Figure S13. Immunofluorescence staining of ICAM-1 (red) and FITC-labeled MPBzyme (green), MPBzyme@NHM (green) and MPBzyme@NCM (green) in the brain sections of mice at $24 \mathrm{~h}$ after injection. Scale bar: $100 \mu \mathrm{m}$. 

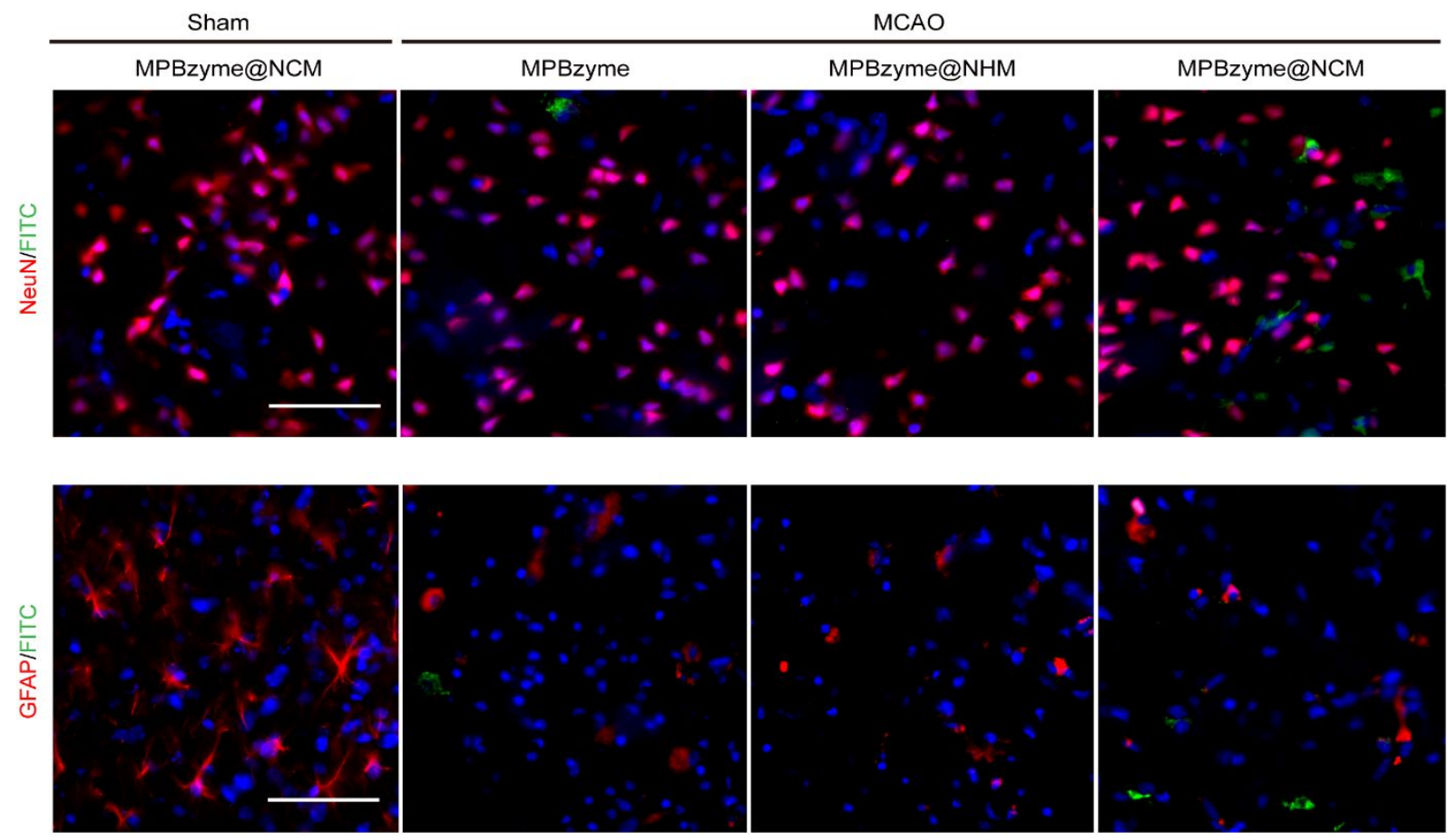

Figure S14. Immunofluorescence staining of NeuN (red), neurons marker, GFAP (red), astrocytes marker, and FITC-labeled MPBzyme@NCM (green), MPBzyme (green) or MPBzyme@NHM (green) in the brain sections at day 3 after injection. Scale bar: $100 \mu \mathrm{m}$.
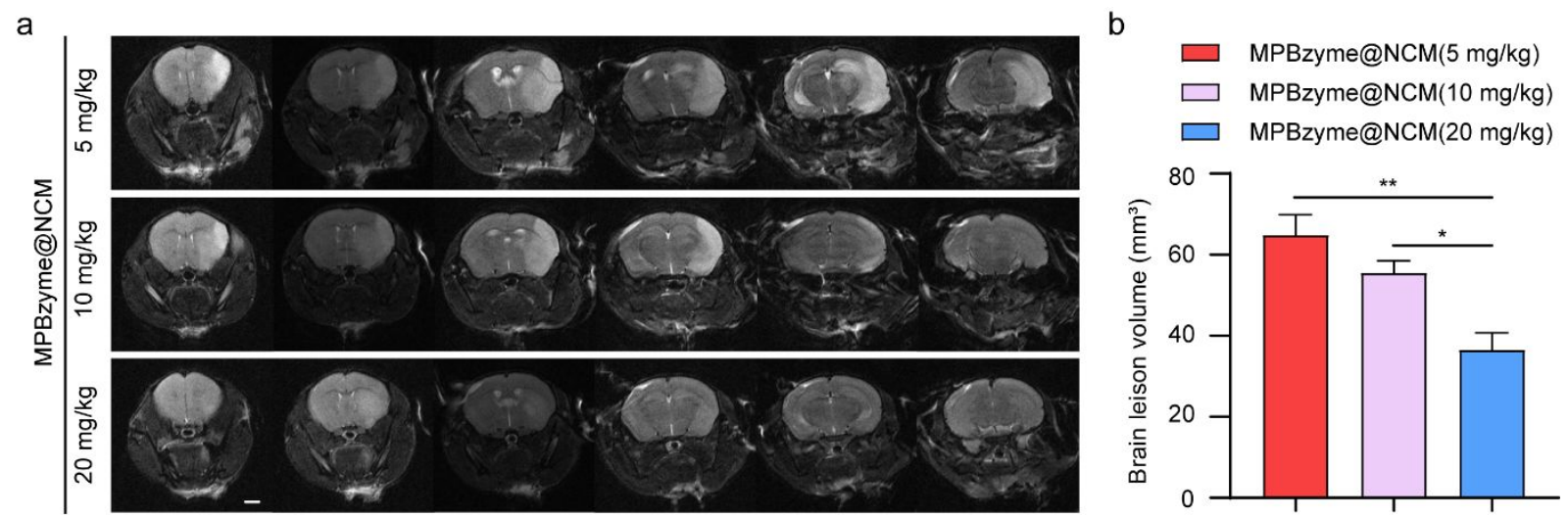

Figure S15. In vivo cerebral MRI of stroke mice at day 3 post-stroke after injection of MPBzyme@NCM of different concentration. (a) Axial view of T2-weighted cerebral MR images of stroke mice. Scale bar: $1 \mathrm{~mm}$. (b) Comparison of brain lesion volume measured in MR images among groups. $n=3$ per group. Data are presented as mean \pm SEM. P values were calculated by one-way ANOVA. $(* \mathrm{p}<0.05$ and $* * \mathrm{P}<0.01)$. 


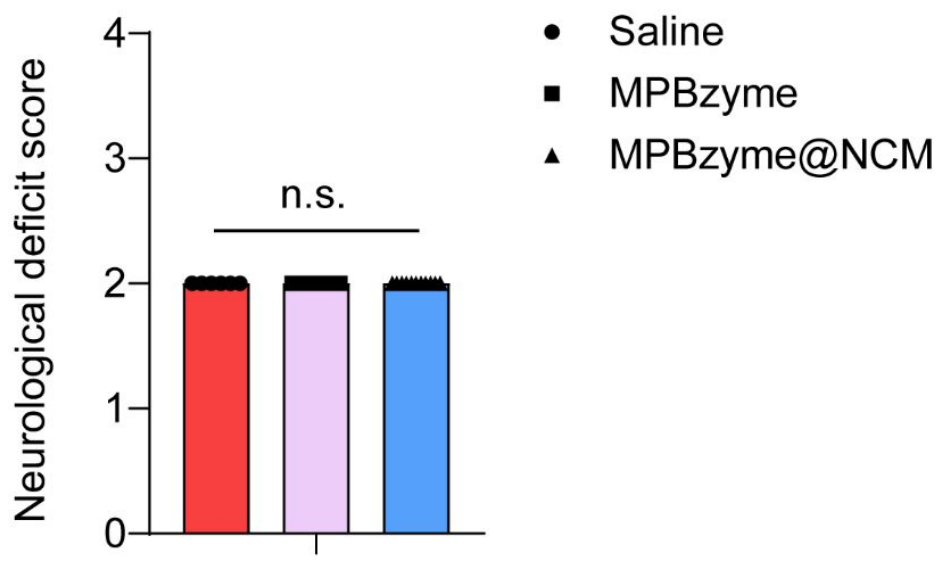

Figure S16. Comparison of neurological deficit score at 1 h after surgery among groups. $n=6$ 11 per group. Data are presented as mean \pm SEM. $\mathrm{P}$ values were calculated by one-way ANOVA. (n.s., not significant).

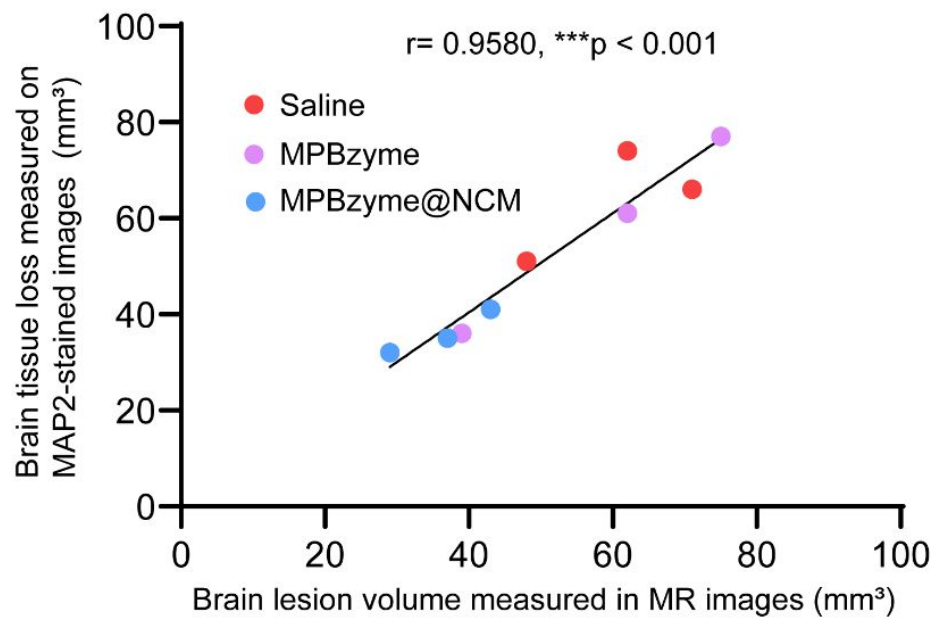

Figure S17. Correlation of brain lesion volume measured in MR images to brain tissue loss measured on MAP-2 stained images. $n=3$ per group. $\mathrm{P}$ value was calculated by two-tailed Pearson linear correlation analysis. 

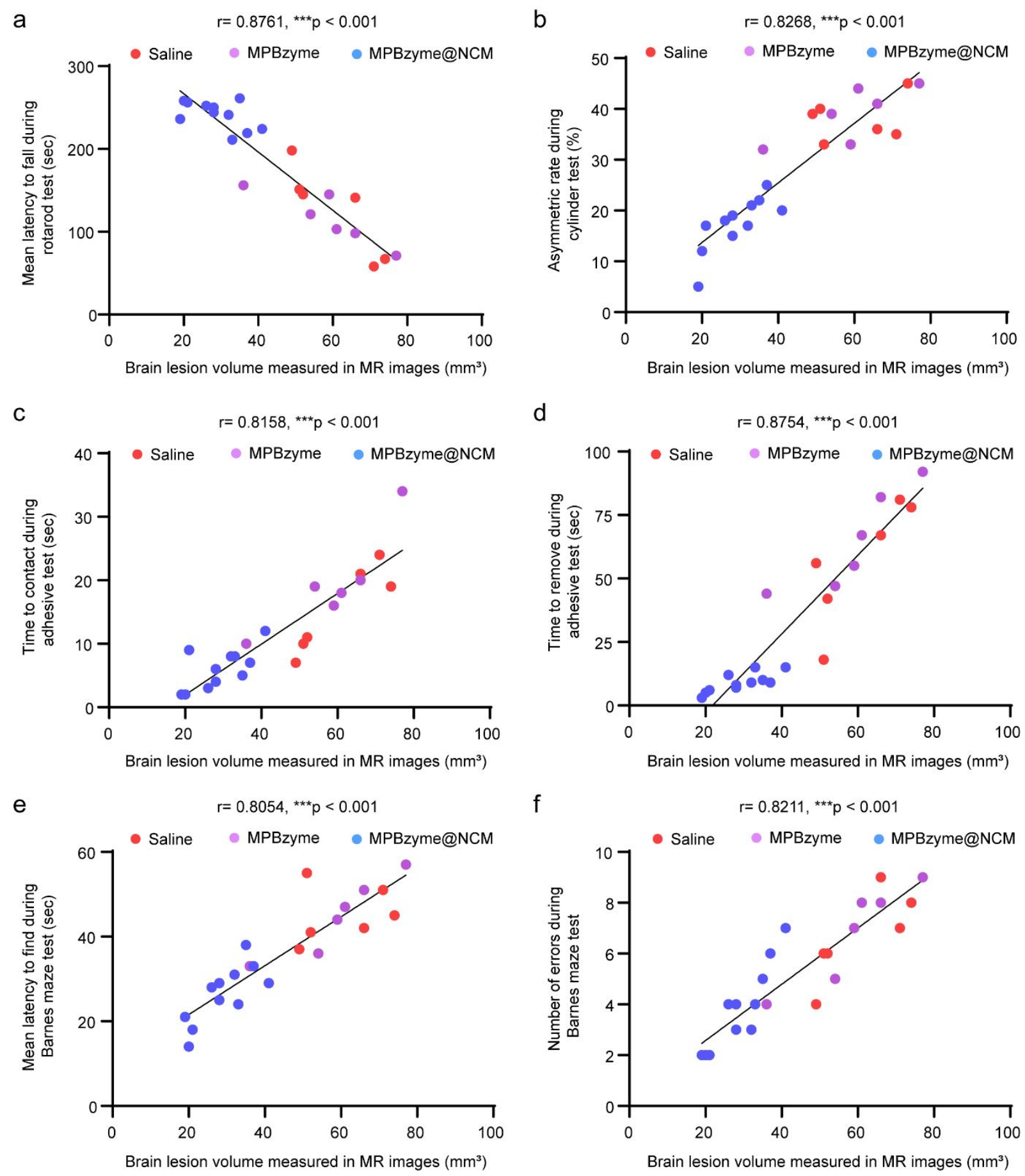

Figure S18. Correlation between brain lesion volume measured in MR images and mean latency to fall during rotarod test (a), asymmetric rate during cylinder test (b), time to contact (c) and time to remove (d) during adhesive test, mean latency to find (e) and number of errors (f) during Barnes maze test at day 28 after stroke. $\mathrm{n}=6-11$ per group. $\mathrm{P}$ value was calculated by two-tailed Pearson linear correlation analysis. 
a

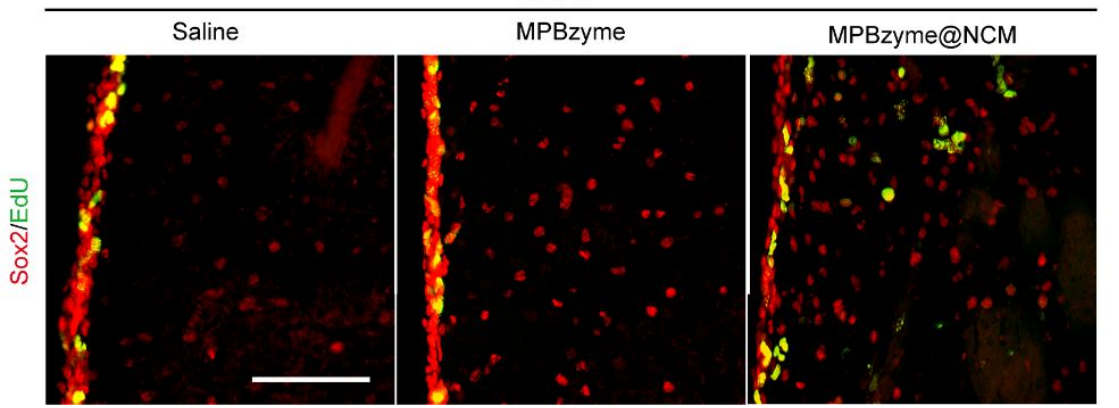

b

$\square$ Saline $\square$ MPBzyme $\square$ MPBzyme@NCM

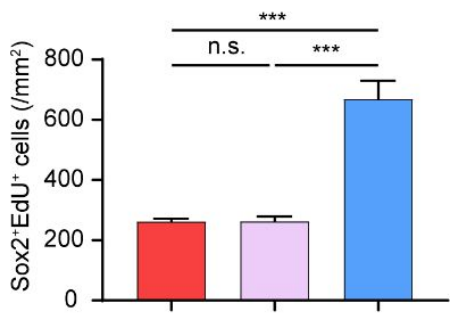

Figure S19. In vivo up-regulation of neurogenesis at day 14 after stroke by MPBzyme@NCM.

(a) Double immunofluorescence staining of Edu with Sox2 in subventricular zone (SVZ) of mice with different treatment at day 14 after stroke. Scale bar: $100 \mu \mathrm{m}$. (b) Comparison of Sox $2^{+} /$EdU $^{+}$cells in SVZ among groups. $\mathrm{n}=3$ per group. Data are presented as mean $\pm \mathrm{SEM}$. $\mathrm{P}$ values were calculated by one-way $\operatorname{ANOVA}(* * * \mathrm{P}<0.001$, n.s., not significant).

a

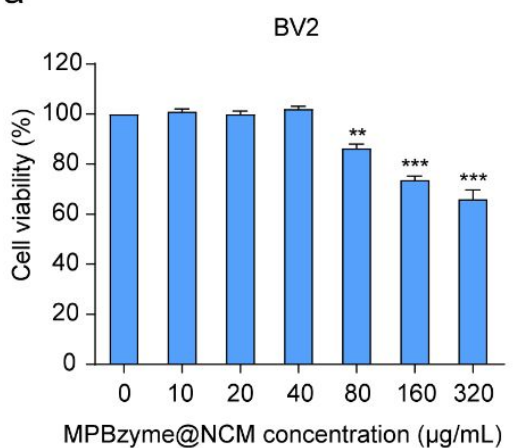

b

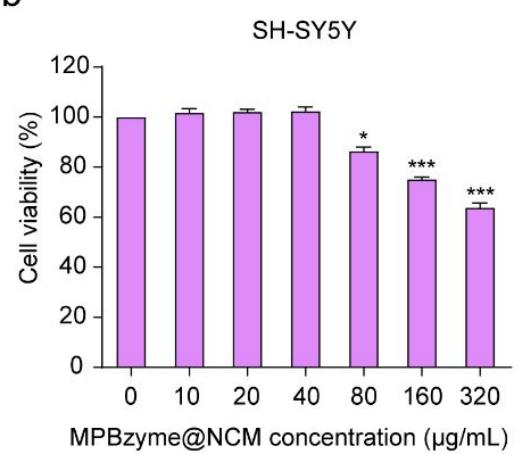

C

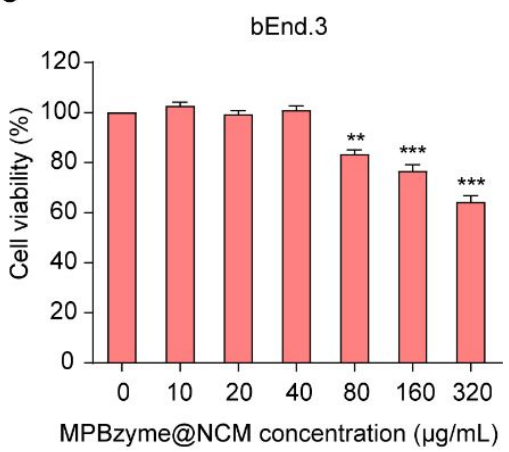

Figure S20. Cell viability of BV2 cells (a), SH-SY5Y cells (b), and bEnd3 cells (c) after 24 h incubation with different concentration of MPBzyme@NCM. n = 3 per group. Data are presented as mean \pm SEM. $\mathrm{P}$ values were calculated by one-way ANOVA $\left(* \mathrm{P}<0.05,{ }^{*} \mathrm{P}<\right.$ $0.01, * * * \mathrm{P}<0.001$, v.s.no MPBzyme@NCM treatment group). 


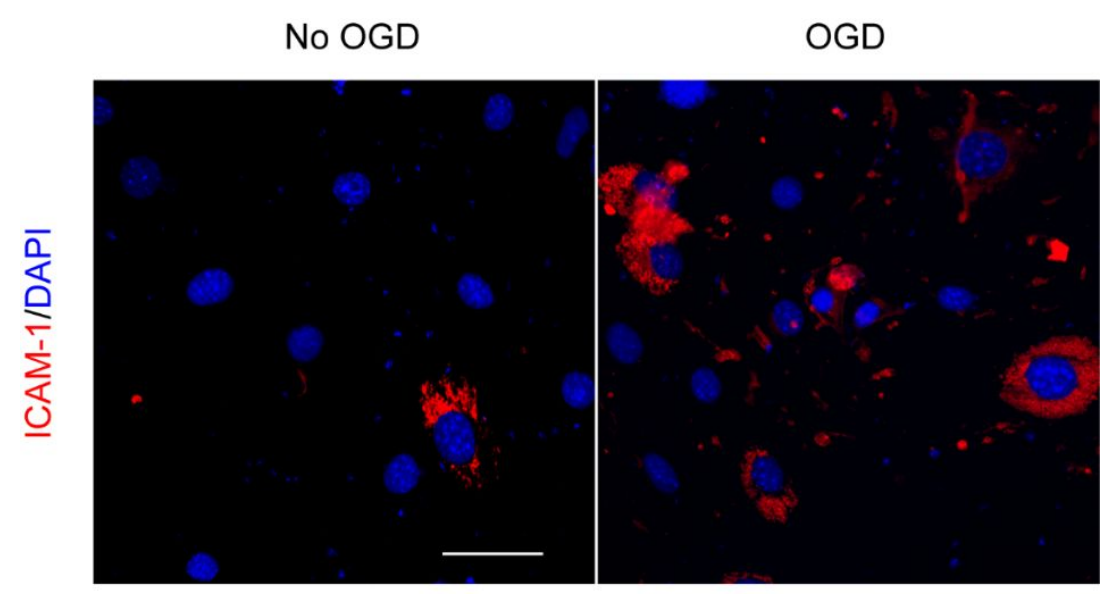

Figure S21. Immunofluorescence staining of ICAM-1 (red) in bEnd.3 cells. Scale bar: $50 \mu \mathrm{m}$.

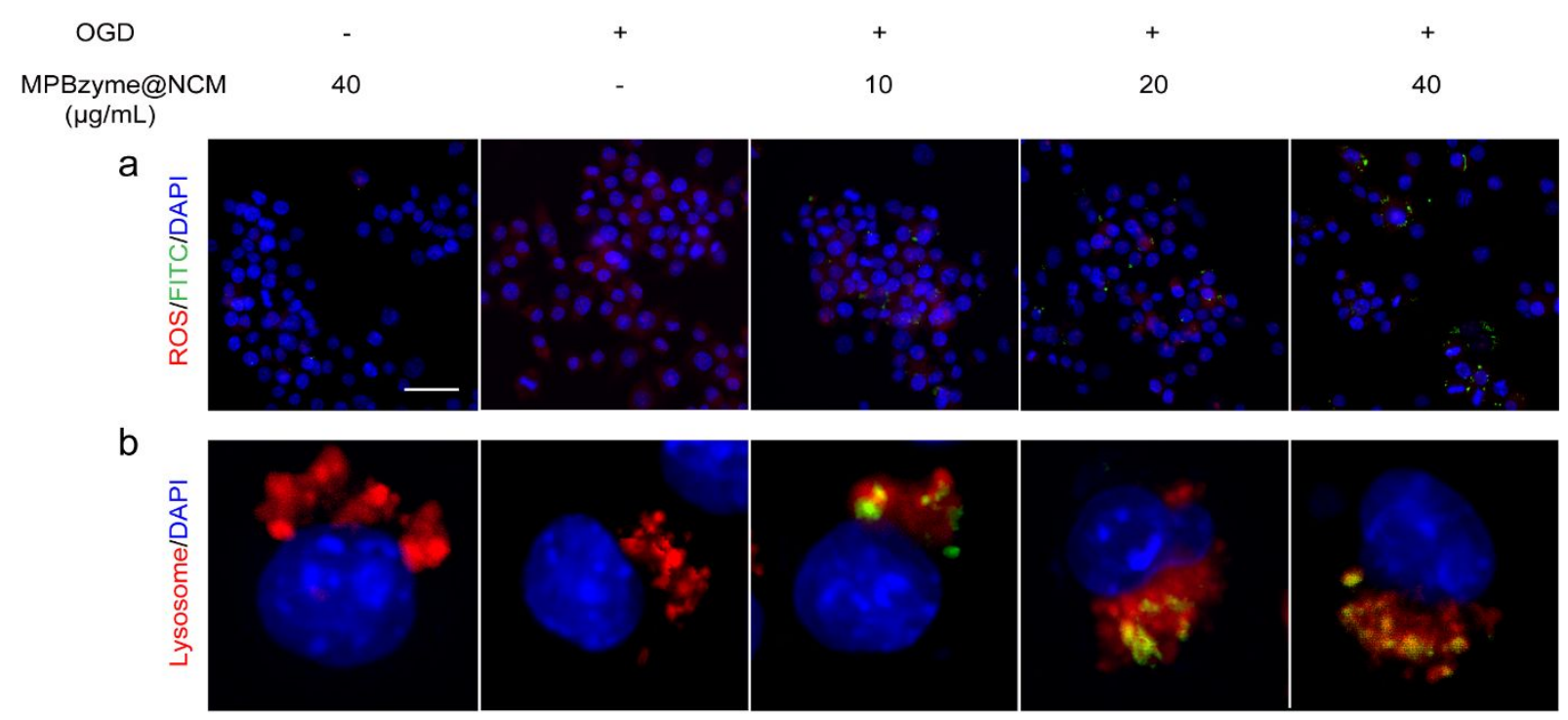

Figure S22. Immunofluorescence staining of DHE (red) with FITC-labeled MPBzyme@NCM (green) in BV2 cells after coculture of bEnd.3 cells and BV2 cells in a transwell system (a). Scale bar: $100 \mu \mathrm{m}$. Localization of MPBzyme@NCM in BV2 cells after coculture of bEnd.3 cells and BV2 cells in a transwell system (b). 


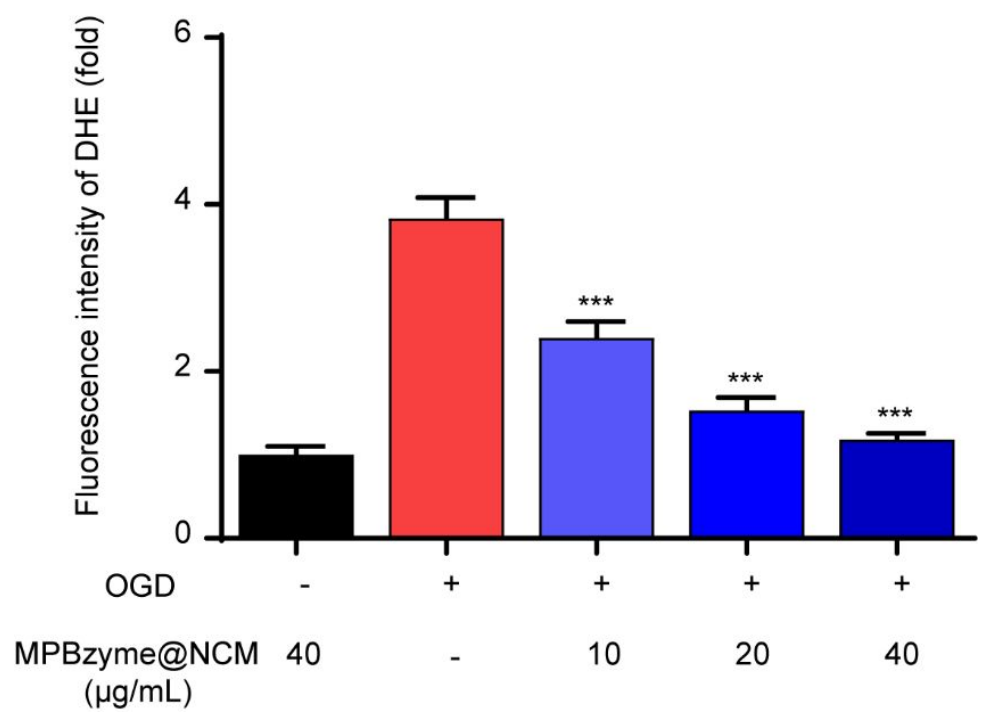

Figure S23. Comparison of the fluorescence intensity of DHE in BV2 cells after different treatment. Data are presented as mean \pm SEM. $\mathrm{n}=3$ per group. $\mathrm{P}$ values were calculated by one-way ANOVA (*** $\mathrm{P}<0.0001$, v.s. OGD/R treatment).

$$
\begin{aligned}
& \text { a } \\
& \underset{(\mu \mathrm{g} / \mathrm{mL})}{\mathrm{MPBzyme} @ \mathrm{NCM}}
\end{aligned}
$$

C

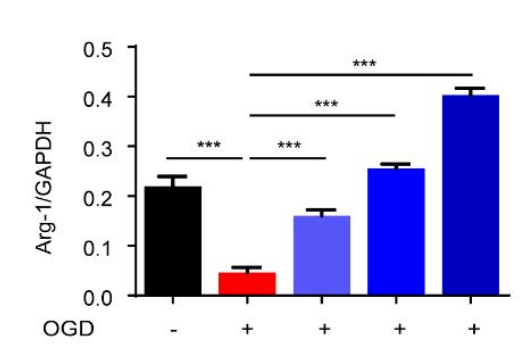

d

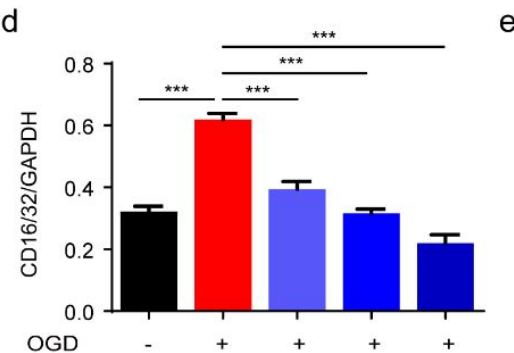

b

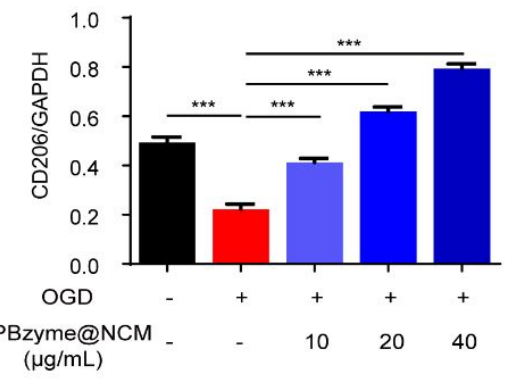

e

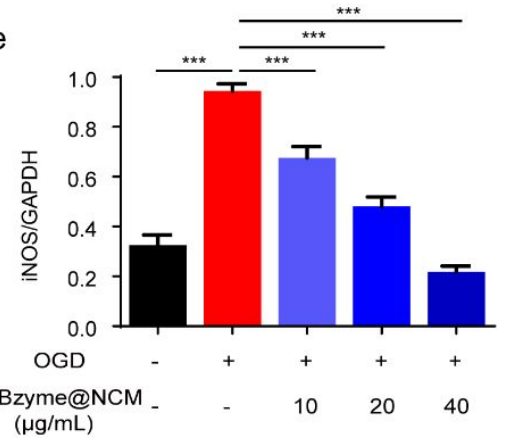

Figure S24. Western blotting analyses for the expressions of M1 and M2 markers in BV2 cells after different treatments (a). Comparison of the protein expression level of CD206 (b), Arg-1 (c), CD16/32 (d) and iNOS (e) among groups. $n=3$ per group. Data are presented as mean \pm SEM. P values were calculated by one-way ANOVA $(* * * \mathrm{P}<0.001)$. 
a

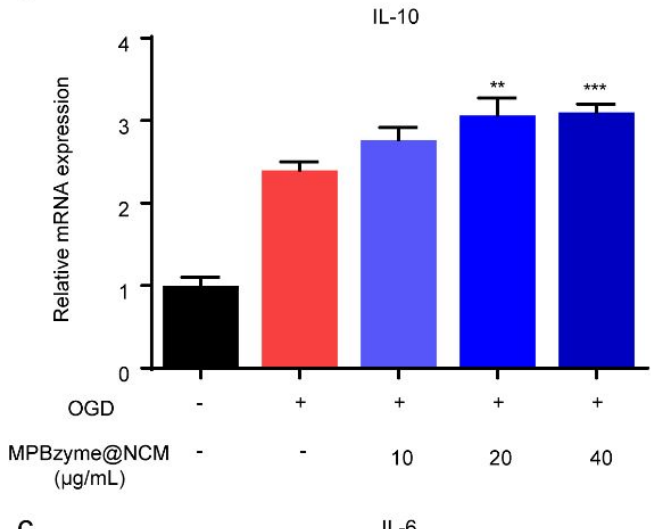

C

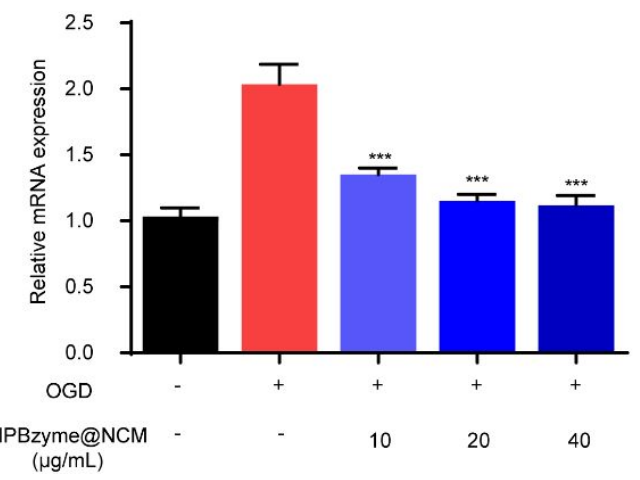

b

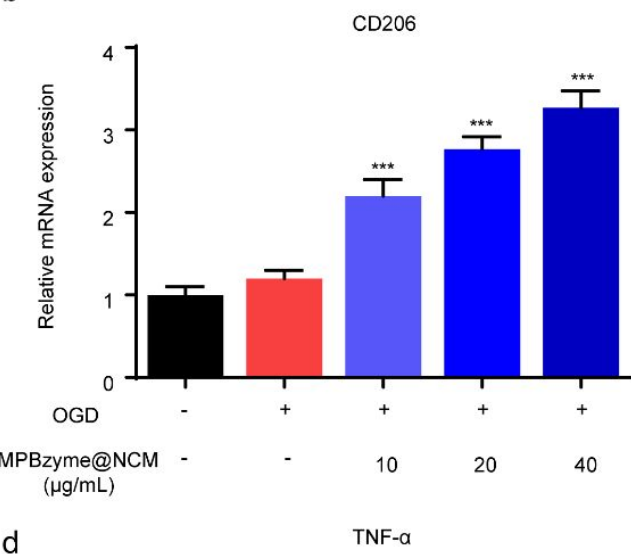

d

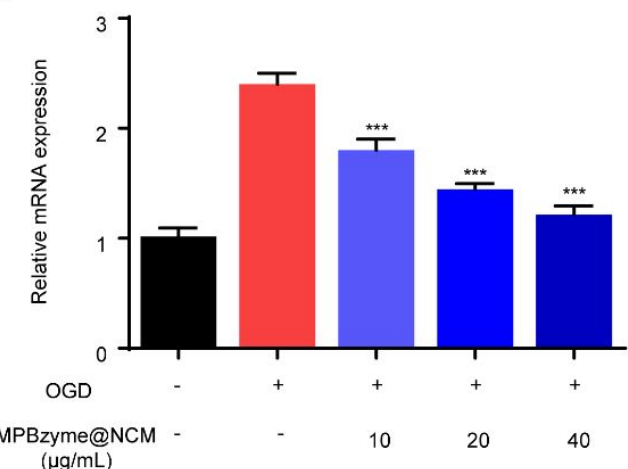

Figure S25. Comparison of the relative mRNA expression of IL-10 (a), CD206 (b), IL-6 (c) and TNF- $\alpha(\mathrm{d})$ in BV2 cells among groups. Data are presented as mean \pm SEM. $\mathrm{n}=3$ per group. $\mathrm{P}$ values were calculated by one-way ANOVA $(* * \mathrm{p}<0.01$ and $* * * \mathrm{P}<0.001$, v.s. OGD treatment). 


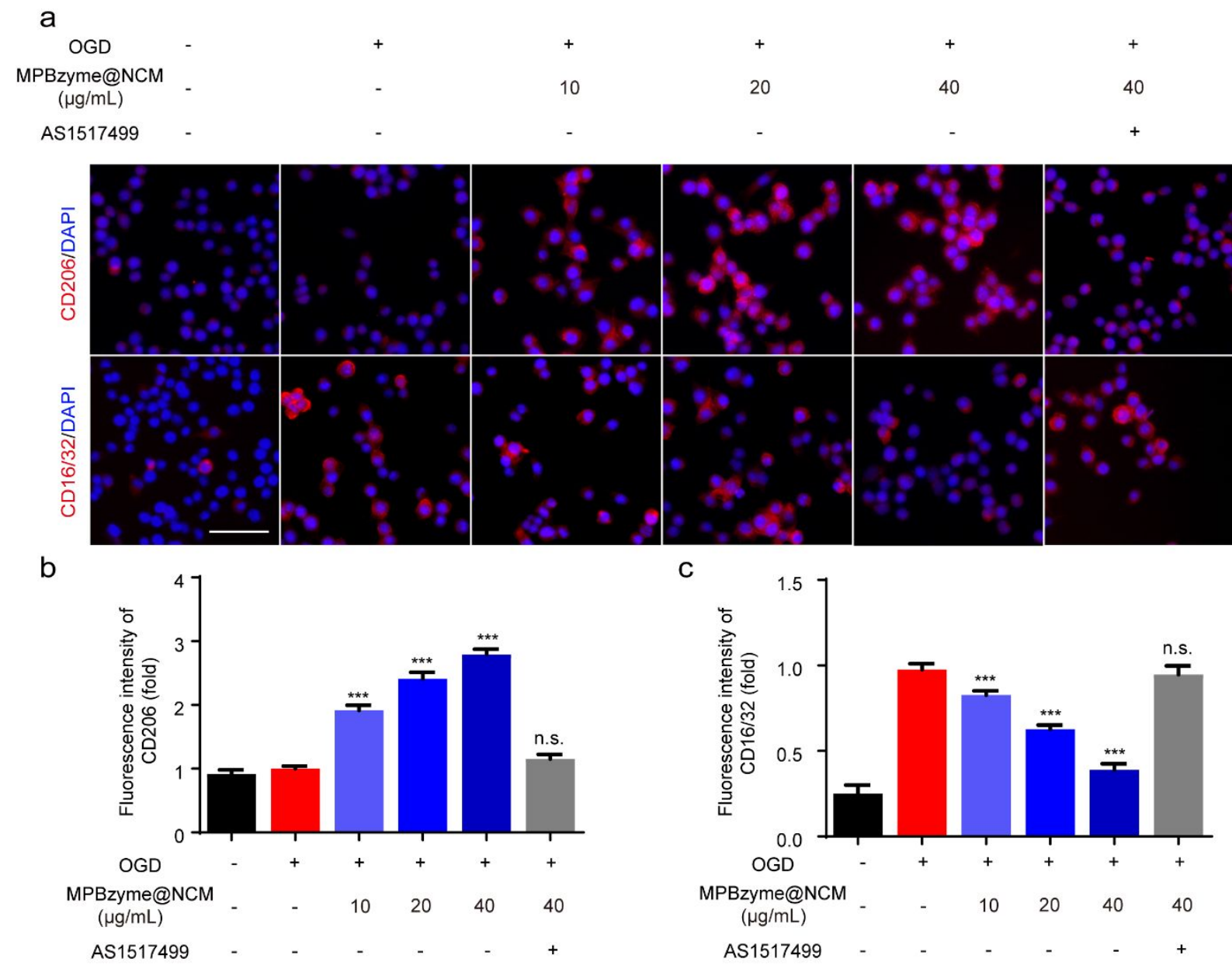

Figure S26. Immunofluorescence staining of CD206 (red) and CD16/32 (red) in BV2 cells. Phenotypic polarization of microglia from M1 to M2 after MPBzyme@NCM treatment was presented in BV2 cells. $\mathrm{n}=3$ per group. Data are presented as mean $\pm \mathrm{SEM}$. P values were calculated by one-way ANOVA (*** $\mathrm{P}<0.0001$, v.s. OGD/R treatment, n.s., not significant). Scale bar: $100 \mu \mathrm{m}$.

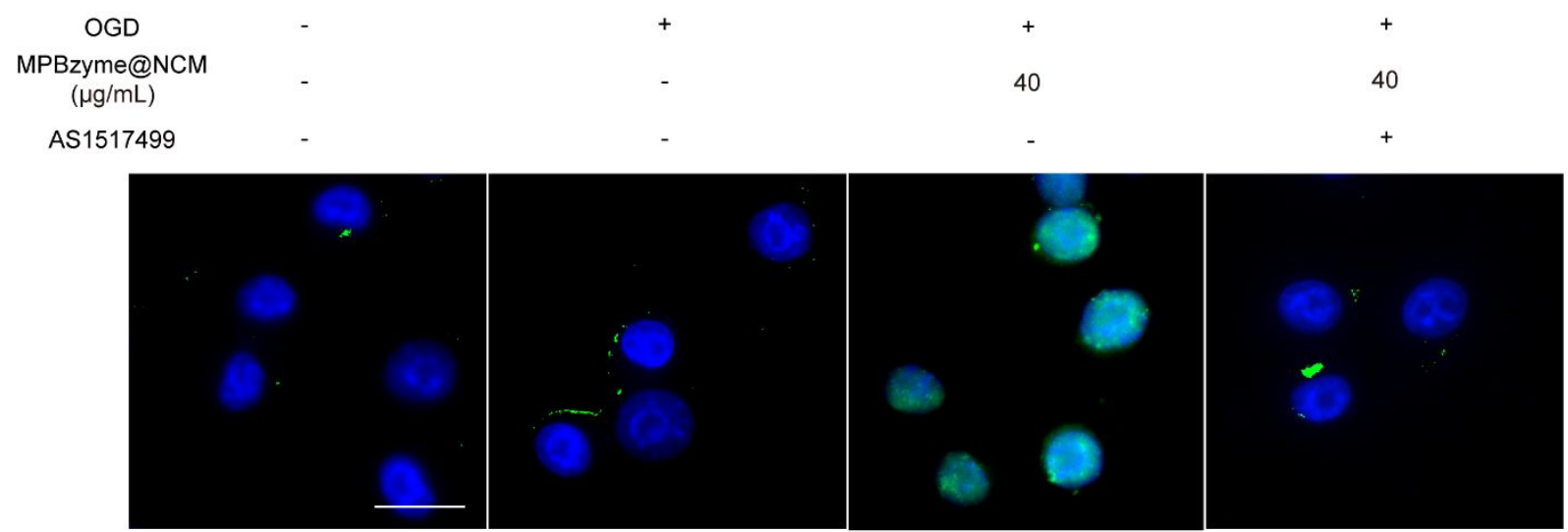


Figure S27. Immunofluorescence staining of p-STAT6 (green) in BV2 cells from differenttreated group. Scale bar: $25 \mu \mathrm{m}$.

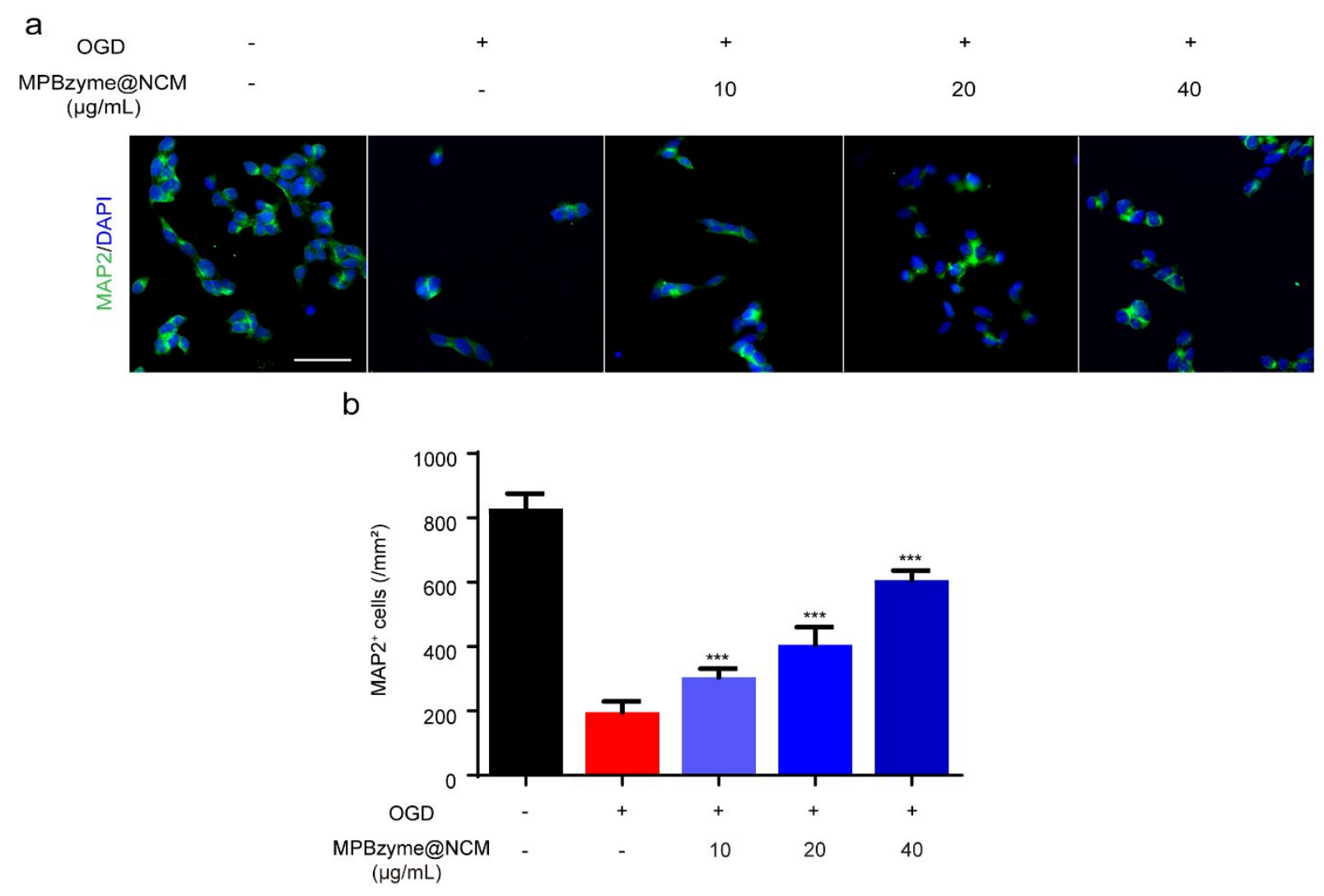

Figure S28. Immunofluorescence staining of MAP2 (green) in SH-SY5Y cells and comparison of numbers of $\mathrm{MAP}^{+}$cells among different groups. $\mathrm{n}=3$ per group. Data are presented as mean \pm SEM. Scale bar: $100 \mu \mathrm{m}$. P values were calculated by one-way ANOVA $(* * * \mathrm{P}<$ 0.0001, v.s. OGD/R treatment, n.s., not significant). 


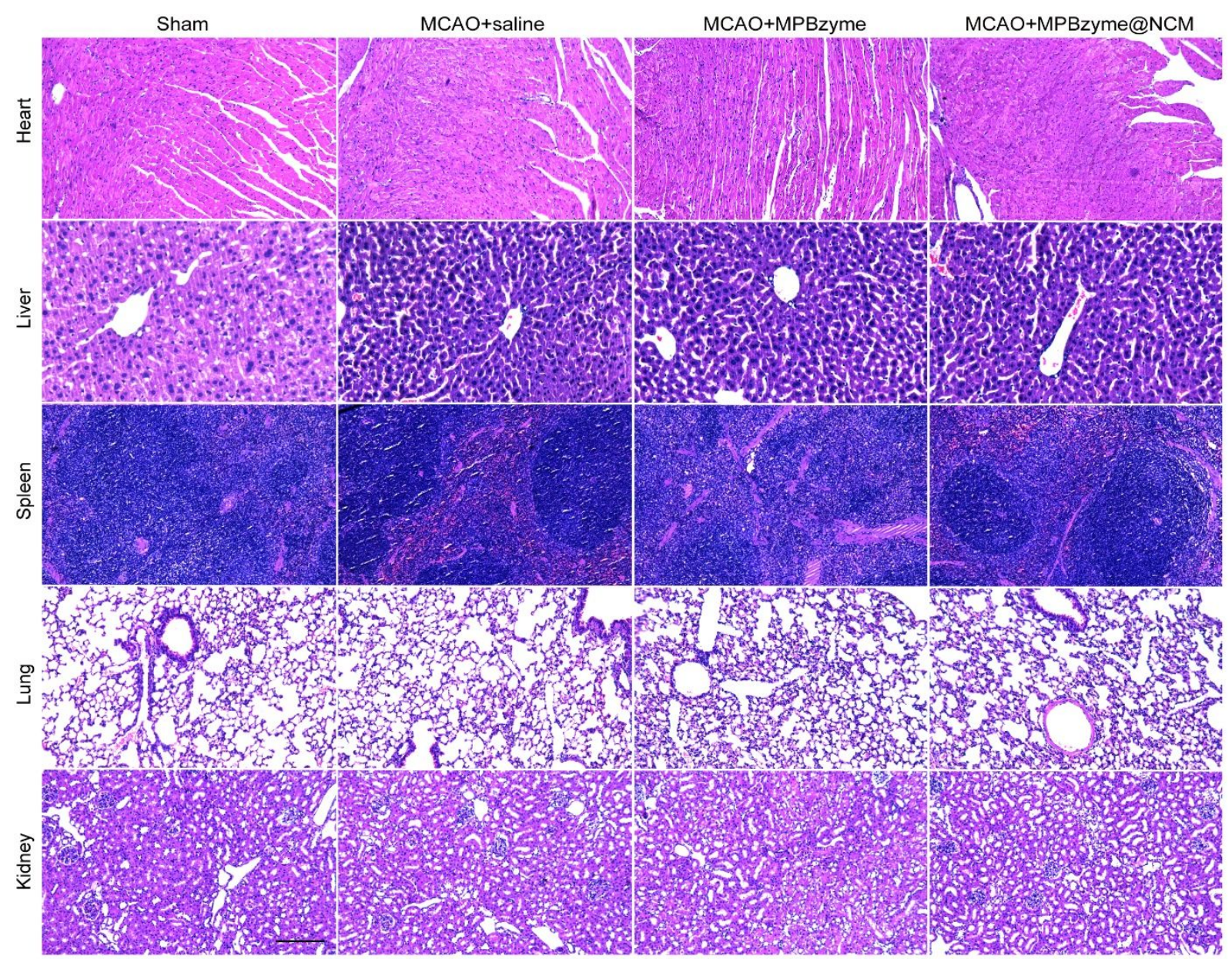

Figure S29. HE staining of main organs from different groups. Scale bar: $200 \mu \mathrm{m}$.

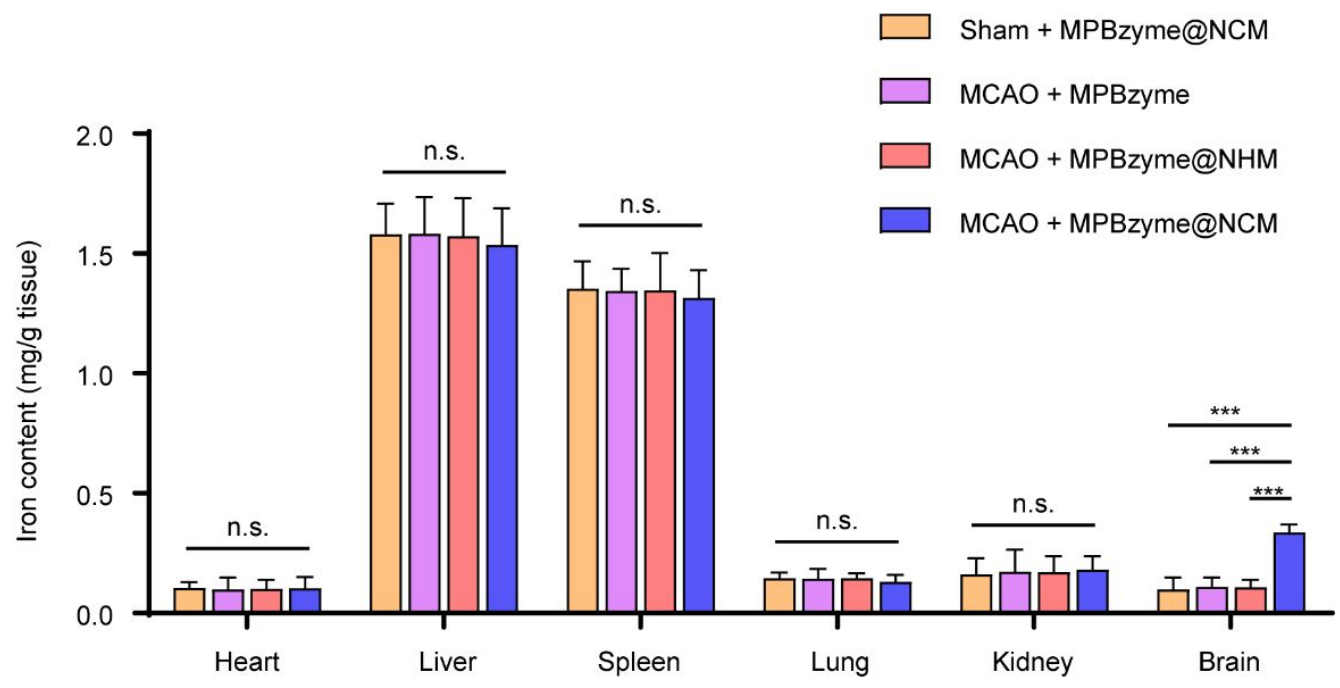

Figure S30. The iron content of main organs from mice after different treatment examined by ICP-OES at $24 \mathrm{~h}$ after injection. $\mathrm{n}=3$ per group. Data are presented as mean $\pm \mathrm{SEM}$. $\mathrm{P}$ values were calculated by one-way ANOVA (*** $\mathrm{P}<0.001$, n.s., not significant). 


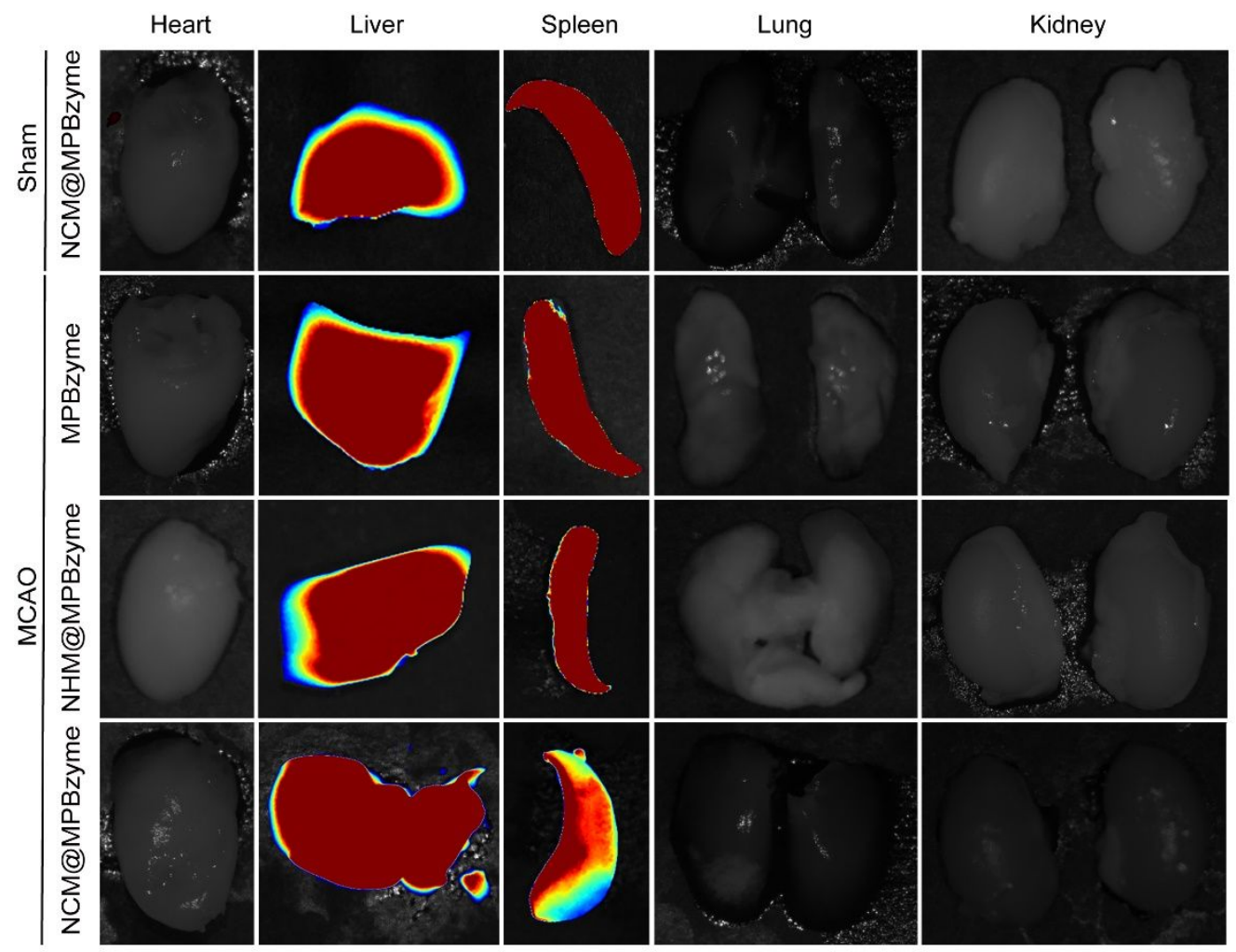

Figure S31. Fluorescence imaging of main organs from different groups at $24 \mathrm{~h}$ after injection of FITC-labeled MPBzyme@NCM, MPBzyme@NHM and MPBzyme. 